\title{
Sample Management: Recommendation for Best Practices and Harmonization from the Global Bioanalysis Consortium Harmonization Team
}

\author{
Michael J. Redrup, ${ }^{1}$ Harue Igarashi, ${ }^{2}$ Jay Schaefgen, ${ }^{3}$ Jenny Lin, ${ }^{4,10}$ Lisa Geisler, ${ }^{5}$ Mohamed Ben M'Barek, ${ }^{6}$ \\ Subramanian Ramachandran, ${ }^{7}$ Thales Cardoso, ${ }^{8}$ and Vera Hillewaert ${ }^{9}$
}

Received 2 June 2015; accepted 7 January 2016; published online 28 January 2016

\begin{abstract}
The importance of appropriate sample management in regulated bioanalysis is undeniable for clinical and non-clinical study support due to the fact that if the samples are compromised at any stage prior to analysis, the study results may be affected. Health authority regulations do not contain specific guidance on sample management; therefore, as part of the Global Bioanalysis Consortium (GBC), the A5 team was established to discuss sample management requirements and to put forward recommendations.
\end{abstract}

The recommendations from the team concern the entire life span of the sample and include the following:

1. Sampling procedures should be described in the protocol or within the laboratory manual. This information should include the volume of the sample to be collected, the required anticoagulant, light sensitivity, collection and storage containers, and labeling with a unique identifier.

2. The correct procedures for processing and then storing the samples after collection at the clinical/non-clinical testing site and during shipment are also very important to ensure the analyte(s) stability and should be documented.

3. Chain of custody for the samples must be maintained throughout the complete life span of each sample. This is typically maintained via paper and electronic data systems, including Laboratory Information Management Systems (LIMS) where available.

4. Pre- and post-analysis storage location and conditions must also be clearly defined at the analytical laboratory. The storage temperature of the samples must be traceable and controlled by monitoring and warning alerts. The team suggests moving away from using temperatures and to adopt standard terminology of "room temperature," "refrigerator," "freezer," and "ultrafreezer" that have defined and industry-wide accepted temperature ranges.

5. At the end of the study, documentation of the samples' disposal is required.

KEY WORDS: LIMS; sample collection; sample disposal; sample management; sample storage.

\section{INTRODUCTION}

Sample management in regulated bioanalysis is arguably one of the most important, and most challenging, aspects for

\footnotetext{
$\overline{{ }^{1} \text { Quotient Bio Analytical Sciences (an LGC company), Newmarket, }}$ UK.

${ }^{2}$ GlaxoSmithKline K. K., Tokyo, Japan.

${ }^{3}$ PPD, Middleton, Wisconsin, USA.

${ }^{4}$ CMIC, Inc, 2860 Forbs Avenue, Hoffman Estates, Illinois 60192, USA.

${ }^{5}$ Covance, Madison, Wisconsin, USA.

${ }^{6}$ NIBR, Novartis Pharma AG, Basel, Switzerland.

${ }^{7}$ Clinigene International Ltd (a Syngene company), Bangalore, India.

${ }^{8}$ Waters Technologies do Brasil, Barueri, Brazil.

${ }^{9}$ Jansen R\&D, Beerse, Belgium.

${ }^{10}$ To whom correspondence should be addressed. (e-mail: jennylin@cmicgroup.com; )
}

clinical and non-clinical study support due to the fact that if the samples are compromised at any stage prior to analysis, the study results can be affected. The analyte concentrations in clinical and non-clinical biological samples are ensured by storage and analysis under conditions that maintain stability through each step of the process. A chain of custody for individual samples is required to document that it was analyzed under conditions that ensured analyte stability and handled in an appropriate manner. However, the regulations (1-8) do not contain specific guidance on the details of sample management; therefore, as part of the Global Bioanalysis Consortium (GBC), the A5 team was established to discuss sample management requirements and put forward recommendations. The team consisted of representatives from North America, South America, Europe, and Asia included members from pharmaceutical companies and contract research organizations. 
This article discusses the various stages of sample management from collection to disposal for biological fluids collected with conventional practices. Recent sampling techniques, such as dried blood spot (DBS) and microsampling, are out of the scope of this article. Recommendations to ensure stability of the analyte in each matrix have already been reported (9); therefore, this manuscript focuses on how to ensure the chain of custody of each sample.

The views of this article are a consensus of the group, but individuals may not agree on all of the points discussed.

\section{SCOPE}

The scope of team A5 included all aspects of sample management from collection to disposal as follows:

- Collection, handling, and storage at the clinical/non-clinical site

- Storage and shipment from clinical/non-clinical testing site to the analytical laboratory, including transitioning through an intermediary facility (e.g., central lab)

- Pre-analysis storage at the analytical laboratory

- Post-analysis storage or shipment

- Disposal

\section{SAMPLE COLLECTION AT CLINICAL/NON- CLINICAL TESTING SITE}

All procedures must be performed as described in the protocol, and if more details are required, supplementary information within a Laboratory Manual and/or Standard Operating Procedure (SOP) should be used. Procedural descriptions that need to be considered include sample volume to be collected, appropriate collection and storage containers, the correct anticoagulant, centrifugation parameters (e.g., time, centrifugal force $[\times g]$, temperature), special conditions (e.g., protection from light), treatment with stabilizers (if required), and the stability of the analyte in the defined conditions. All samples must be labeled clearly with a unique identity. Handwritten labels should not be used. At a minimum, the following labeling information is advised: protocol number, subject/animal number, visit/time, matrix, and a unique identifier (e.g., accession number). In clinical studies, the subject's name or personal information that could be used to identify the volunteer must not be used.

If adequate sample volume collection is permitted, it is advised that samples are split into two (2) portions (e.g., set 1 and set 2). It is also recommended that a quality control process be in place at the collection site to ensure that the procedure described in the protocol and the supplementary information is followed. Any discrepancies must be reported immediately and notification sent to the appropriate personnel.

Appropriate safety procedures need to be in place for potentially hazardous samples such as those containing radioactivity and/or pathogens.

\section{RECORD OF LOCATION AND STORAGE CONDITIONS}

Chain of custody must be maintained during the life span of each sample. This requires a record of the location and storage conditions of the samples at all times at each facility including the time spent in and out of the storage units, time during analysis, and the number of times the sample has been thawed. It is recommended that, when available, a Laboratory Information Management System (LIMS) be used at each facility as this will include an audit trail.

\section{STORAGE AND SHIPMENT FROM CLINICAL/NON- CLINICAL TESTING SITES TO THE ANALYTICAL LABORATORY}

Samples must be stored under conditions in which the analytes are known to be stable and, if stability is not known, under expected stable conditions (e.g. by using stability information for the class of compounds). In the majority of cases, the samples should be refrigerated or frozen as soon as the procedure allows (e.g., immediately after centrifugation for plasma and serum). The storage location and conditions must be recorded from the time of collection until they are shipped. Any discrepancies from the expected storage conditions must be reported immediately to the appropriate personnel.

The sample shipment inventory must be prepared, included within every shipment, and ideally sent electronically to the analytical laboratory or testing facility. At a minimum, the following inventory information is advised: protocol number, subject/animal number, visit/time, matrix, anticoagulant, analyte, set $1 /$ set 2 , and the unique identifier (e.g., accession number).

Shipment of samples must be carried out under conditions where the analytes are known to be stable (or at least expected if stability is not known). Typically this will be on dry ice, refrigerated (wet ice packs), or at ambient temperatures. If shipments are to be transported at ambient temperature, consideration of the local conditions during transit is essential (e.g., assessment of the possible weather conditions during the entire transit between facilities). If samples are checked during transit by the courier, this should be documented along with any action taken (e.g., replenishment of dry ice).

The contact at the analytical laboratory must be notified of the sample shipment with a projected date and time of delivery. A sample inventory must be received at the same time or prior to the arrival of the shipment. If set 1 and set 2 aliquots have been taken, they should not be shipped in the same package to insure the integrity of one aliquot in case of unexpected shipping issues. This includes the possible shipping of the sets on consecutive days.

For sample shipment with expected durations of more than $24 \mathrm{~h}$, temperature data loggers should be considered. Appropriate safety procedures need to be in place for potentially hazardous samples such as those containing pathogens (8) and/or radioactivity. 


\section{STORAGE AT THE ANALYTICAL LABORATORY (PRE- AND POST-ANALYSIS)}

Upon receipt at any facility, the shipper should be notified. The packaging of the samples should be inspected, and there should also be a check that the shipping conditions have been maintained (e.g., plenty of dry ice present, undamaged samples). Any deviations noted should be reported to the responsible personnel at the shipping facility. All samples received must be checked against the sample shipment inventory and those planned within the protocol. Any discrepancies should be communicated to the appropriate personnel at the study site, central lab, and sponsor personnel, including in good laboratory practice (GLP) studies, the study director.

Once checked, the samples should be logged in to the facility's sample tracking system (e.g., LIMS) and placed in storage conditions under which the analytes are known to be stable (if the stability is known). Set 1 and set 2 samples should not be stored in the same unit. The location and storage conditions of samples must be recorded and the responsible personnel for the analysis notified. Transfer from storage to the analyst and return to appropriate storage after the analysis should also be tracked. Quality control samples and calibration standards used during analysis should be stored under the same conditions as the study samples and in appropriate containers and storage units. Samples should be maintained under appropriate conditions until disposal is authorized. Disposal of the samples should also be recorded.

\section{GENERAL STORAGE REQUIREMENTS}

The temperature of all storage units must be monitored and warning alerts put in place for excursions out of a predefined range (e.g., doors left ajar, unit failure). Actions for temperature excursions should be according to predefined SOPs at each site managing the stored samples. A disaster recovery plan must be in place to cover unit failure at any time. This plan should list who should be notified and what action should be taken.

Sample storage areas need to be secure, fit-for-purpose, and able to continually maintain the required storage conditions. Temperature mapping should be considered for large refrigerators or freezers, during which the temperature should be checked at multiple points within the unit. Refrigerators and freezers must be maintained according to any manufacturer recommendations, and ice should not be allowed to excessively accumulate.

\section{PROPOSED STORAGE TEMPERATURES}

The storage conditions between clinical/non-clinical testing sites and analytical laboratories often appear to differ slightly in temperature settings depending on the historical experiences, SOP requirements at each site, or the performance of the units. For example, a freezer set at $-80^{\circ} \mathrm{C}$ in analytical laboratory may actually be operating at a higher temperature of $-72^{\circ} \mathrm{C}$. Additionally, a similar freezer set at
Table I. Proposed Storage Condition Nomenclature

\begin{tabular}{llll}
\hline Conditions & Set point ${ }^{\circ} \mathrm{C}$ & Minimum ${ }^{\circ} \mathrm{C}$ & Maximum ${ }^{\circ} \mathrm{C}$ \\
\hline Room temp & - & 10 & 30 \\
Refrigerator & 5 & 2 & 8 \\
Freezer & -20 & -30 & -15 \\
Ultra-freezer & -75 & -85 & -65 \\
\hline
\end{tabular}

$-70^{\circ} \mathrm{C}$ at a non-clinical testing site (another facility) may actually be operating at a $7 \%$ lower temperature $\left(-77^{\circ} \mathrm{C}\right)$. In these cases, the freezer set at a nominal temperature of $-70^{\circ} \mathrm{C}$ is actually colder than a freezer set at $-80^{\circ} \mathrm{C}$. These temperature differences do not typically affect the stability of the analyte(s) in the sample because the specimen status is not altered. However, problems in the documentation of the storage temperatures exist. In these examples, the analytical laboratory has documented "stability data at $-80^{\circ} \mathrm{C}$," whereas the non-clinical testing site may have stored the samples at " $-70^{\circ} \mathrm{C}$ " prior to shipment. Both facilities would have been operating appropriately and in accordance with their SOPs, with very little difference in the actual storage temperature of the samples. However, the conditions and documentation are not aligned, and additional stability testing may be considered to be needed.

To steer away from this formalistic issue, it is proposed that the industry moves away from using temperatures to define the storage conditions and adopt standard naming terminology for four main storage conditions: "room temperature," "refrigerator," "freezer," and "ultra-freezer" as detailed below in Table I along with proposed acceptable ranges for these conditions.

\section{SAMPLE DISPOSAL}

Samples should be disposed of after the final report is issued or when required by directives given in the informed consent paperwork for clinical studies. In non-clinical GLP studies, disposal may be defined within the protocol and, when not, in all cases is at the discretion of the study director. In clinical studies, a volunteer has given consent to what can be done with their donated material and can change this at any time. Therefore, it is critical to follow their directives, and any changes (e.g. withdrawal of consent), and dispose of samples when required. The analytical laboratory should document that the samples have been disposed of at the time of disposal.

\section{CONCLUSION}

The team has put forward a number of recommendations to ensure sample integrity through sample management. The recommendations cover the entire life span of each sample including collection in the clinical/nonclinical testing site, transportation, analysis, storage, and disposal. 
The main recommendations include clearly defining what is needed upfront in the protocol and the lab manual, strict documentation of the chain of custody throughout the life span of each sample, moving away from using temperatures to define storage conditions and adopting new standard terminology of the four main storage conditions, and the disposal process. Essential throughout these processes are sound documentation practices.

With these recommendations, the team feels that the management of clinical and non-clinical samples will be maintained with high fidelity and properly documented.

\section{REFERENCES}

1. US Department of Health and Human Services, Food and Drug Administration, Center for Drug Evaluation and Research, Center for Veterinary Medicine. Draft Guidance for industry: Bioanalytical method validation. 2013.

2. US Department of Health and Human Services, Food and Drug Administration, Center for Drug Evaluation and
Research, Center for Veterinary Medicine. Guidance for industry: Bioanalytical method validation. 2001.

3. European Medicines Agency, Committee for Medicinal Products for Human Use. Guideline on Bioanalytical method validation. 2011.

4. Agencia Nacional de Vigilancia Sanitaria, Brazil. Resolucao RDC No 27. May 2012.

5. Ministry of Health, Labour and Welfare, Japan. Guideline on Bioanalytical Method Validation in Pharmaceutical Development. 2013.

6. Ministry of Health, Labour and Welfare, Japan. Guideline on Bioanalytical Method (Ligand Binding Assay) Validation in Pharmaceutical Development. 2014.

7. European Medicines Agency, GCP Inspectors Working Group. Reflection paper for laboratories that perform the analysis or evaluation of clinical trial samples. 2012.

8. World Health Organization. Guidance on regulations for the Transport of Infectious Substances 2015-2016 http:// apps.who.int/iris/bitstrea m/10665/149288/1/ WHO_HSE GCR 2015.2 eng.pdf?ua $=1 \& u a=1$

9. van de Merbel N, Natasha S, Manish Y, et al. Stability: recommendation for best practices and harmonization from the Global Bioanalysis Consortium Harmonization Team. AAPS J. 2014;16(3):392-9. doi:10.1208/s12248-014-9573-z. 